\title{
Determination of Optimal Conditions for Parthenogenetic Activation and Subsequent Development of Rat Oocytes In Vitro
}

\author{
Eiji MIZUTANI ${ }^{1) \#, ~ J i n-Y i ~ J I A N G ~}{ }^{1,2)}$, Satoshi MIZUNO1), Ikuo TOMIOKA'), \\ Tadahiro SHINOZAWA ${ }^{1)}$, Jin KOBAYASHI ${ }^{3)}$, Hiroshi SASADA ${ }^{1)}$ and Eimei SATO ${ }^{1)}$ \\ 1) Laboratory of Animal Reproduction, Graduate School of Agricultural Science, Tohoku \\ University, Sendai 981-8555, Japan, ${ }^{2)}$ Reproductive Biology Unit, Departments of Obstetrics \\ \& Gynecology and Cellular \& Molecular Medicine, University of Ottawa, Hormones, Growth \\ and Development Program, Ottawa Health Research Institute, The Ottawa Hospital (Civic \\ Campus), Ottawa, Ontario, Canada K1Y 4E9, ${ }^{3)}$ Miyagi Agricultural College, Sendai, Japan \\ \#Present: Laboratory of Genomic Reprogramming, Center for Developmental Biology, RIKEN \\ Kobe Institute, Kobe, Hyogo, Japan
}

\begin{abstract}
The present study was undertaken to determine optimal conditions for parthenogenetic activation and subsequent development of rat oocytes. Oocytes from immature Wistar-Imamichi (WI) and Sprague Dawley (SD) rats were activated by electrical stimulation in combination with 6dimethylaminopurine (6-DMAP) to assess whether different rat strains display different responses to activation treatment. Since the cleavage rates of activated oocytes were significantly higher in WI than SD strain rats, WI rats were used for the subsequent experiments to determine the effects of post-hCG time, culture duration, different activation protocols (electrical stimulation with 6-DMAP or ionomycin with 6-DMAP) and osmolarity of the activation medium on the activation and subsequent development of WI rat oocytes. For oocytes activated by electrical stimulation combined with 6DMAP, the percentages of oocytes that were activated and that developed to blastocysts were higher when oocytes were collected at 18-20 h than at any other time points after hCG injection (16, 22-24 h). Culturing for $2-6 \mathrm{~h}$ before activation treatment markedly decreased the percentage of activated oocytes that developed to beyond the four-cell stage. There were no differences in the percentages of oocytes with pronuclear formation and subsequent development to the two-cell and blastocyst stages between oocytes that were activated by electrical stimulation or ionomycin, both followed by 6-DMAP treatment. Activation of oocytes by ionomycin and 6-DMAP, both in low osmolarity media (246 $\mathrm{mOsM})$, markedly increased the cleavage rates and percentages of high quality blastocysts $(71 \%)$. The optimal conditions determined in the present study with simplified activation protocols and high efficiency of activation and subsequent development of WI rat oocytes will be helpful for further research involving nuclear transfer in the rat.

Key words: Ovulation, Culture duration, Ionomycin, 6-Dimethylaminopurine, Osmolarity, Parthenogenetic activation, Oocyte, Rats
\end{abstract}

(J. Reprod. Dev. 50: 139-146, 2004)

S everal animal species, including sheep, mice, cattle, goats, rabbits, cats, pigs, mules and, horses have been reproduced by somatic cell

Accepted for publication: November 25, 2003

Correspondence: E. Sta (e-mail: eimei@bios.tohoku.ac.jp) nuclear transfer [1]. However, attempts to produce rats by nuclear transfer using various somatic cells including cumulus and fibroblast cells have been unsuccessful [2-6]. Most recently, Zhou et al. succeeded in producing a rat clone [7], but the 
success rates of producing clone animals by nuclear transfer are still very low. Since activation of the recipient oocyte is a crucial step in successful nuclear transfer techniques [8], incomplete activation of reconstructed embryos may in part be responsible for the difficulty of rat nuclear transfer. At present, the available information regarding parthenogenetic activation of rat oocytes is limited. Zernicka-Goetz (1991) reported that rat oocytes could be activated in vitro by chloral hydrate and puromycin, but subsequent development beyond the four-cell stage was not observed even if activated embryos were transplanted into ligated oviducts of female mice [9]. Our recent results showed that $50 \%$ of rat oocytes developed to blastocysts when activation was induced by electrical stimulation combined with 6dimethylaminopurine (6-DMAP) treatment. However, the quality of these blastocysts was poor in terms of morphology and the cell number was low $(26.9 \pm 1.9)$, ranging from 22 to 36 [10]. A more recent study showed that Wistar rat oocytes could be activated by electrical stimulation, ethanol or strontium treatment. However, the efficiency of blastocyst formation was low (12.1-29.6\%) and dramatic variations existed among individual rats in the efficiency of parthenogenetic activation of oocytes and developmental ability of the resulting embryos [8]. These results suggested that research into parthenogenetic activation of rat oocytes was insufficient, and further investigation was needed.

In the present study, to determine the optimal conditions for parthenogenetic activation and subsequent development of rat oocytes, we first assessed the differences of the responses of oocytes from Wistar-Imamichi (WI) and Sprague Dawley (SD) rat strains to activation treatment by electrical stimulation in combination with 6-DMAP. Since the cleavage rates of activated oocytes were significantly higher in the WI than the SD strain, oocytes of WI rats were then used for experiments to determine the effects of post-hCG time, culture duration, different activation protocols (electrical stimulation with 6-DMAP or ionomycin with 6DMAP) and osmolarity of the activation medium on activation and subsequent development. Our results showed that one the activation procedures used here was simple yet effective, as indicated by high percentages of blastocysts with good morphology and large cell numbers.

\section{Materials and Methods}

\section{Preparation of animals}

WI and SD rats were housed in a room at a controlled temperature of $24 \pm 2 \mathrm{C}$ and humidity of $65 \pm 5 \%$ with lights on at 6:00 and off at 18:00. Animals were given bullet-type commercial rat feed [High Pure Ace (P), Itochu Co., Ishinomaki, Japan) and tap water ad libitum, as described previously [10]. The studies were approved by the Ethics Committee for Care and Use of Laboratory Animals for Biomedical Research of the Graduate School of Agricultural Science, Tohoku University.

\section{Oocyte collection}

Sexually immature WI and SD female rats (25-40 days old) were injected intraperitoneally with $10 \mathrm{IU}$ of equine chorionic gonadotrophin (eCG, Teikoku Hormone Mfg, Co., Ltd., Tokyo) followed by intraperitoneal injection of 10 IU of hCG (Sankyo Kabu Company, Tokyo) 48-52 h later. Animals were sacrificed by cervical dislocation $16-26 \mathrm{~h}$ after administration of hCG in the indicated experiments. Cumulus-oocyte cell complexes (COCs) were collected by cutting the oviducts in PBS (-) supplemented with $1 \mathrm{mg} / \mathrm{ml}$ fatty acid-free BSA (Sigma Chemical Co., St. Louis, MO) and treated with $0.1 \%(\mathrm{w} / \mathrm{v})$ hyaluronidase (Sigma). After three washes with fertilization medium [mR1ECM [11-13] containing $110 \mathrm{mM} \mathrm{NaCl}$ (Wako Pure Chemical Industries Ltd, Osaka Japan) and 4 $\mathrm{mg} / \mathrm{ml}$ BSA (fatty acid-free, No. A-6003, Sigma) and omitting polyvinylalcohol], oocytes were transferred to $100 \mu \mathrm{l}$ of fertilization medium.

\section{Oocyte activation by electrical stimulation combined with 6-DMAP}

Oocytes were activated as described previously [10]. Briefly, after washing, oocytes were placed between two wire electrodes (1 mm apart) on a fusion chamber slide with $30 \mathrm{ml}$ of activation solution containing mannitol, $0.1 \mathrm{mM} \mathrm{CaCl}_{2}, 0.1$ $\mathrm{mM} \mathrm{MgSO}_{4}$ (all from Wako) and fatty acid-free BSA (Sigma). A DC pulse of $100 \mathrm{~V} / \mathrm{mm}$ was then applied to the oocytes twice at intervals of $1 \mathrm{sec}$ for a duration of $99 \mu \mathrm{sec}$. After the electrical stimulation, the oocytes were treated in fertilization medium containing $2 \mathrm{mM}$ 6-DMAP for $4 \mathrm{~h}$. After activation, the oocytes were transferred to $100 \mu \mathrm{l}$ of fertilization medium and cultured for 22-24 h. They were then transferred to mR1ECM medium 
and culturing was continued under $5 \% \mathrm{CO}_{2}$ in air at $37 \mathrm{C}$.

\section{Experimental design}

Experiment 1 was designed to test the response of oocytes from two rat strains to the activation treatment applied. Oocytes were collected from WI and SD rats 18 hours after hCG injection, and activated by electrical stimulation combined with 6DMAP as described above. The oocytes were then cultured in fertilization medium for 22-24 $\mathrm{h}$ and in mR1ECM medium for a further 4 days. Three replicate experiments were performed. Based on the results of this experiment, oocytes of WI rats were used for the subsequent experiments.

Experiment 2 was conducted to test the effect of post-ovulation duration on parthenogenetic activation of rat oocytes in vitro. Oocytes from WI rats were collected $16,18,20,22,24$ or $26 \mathrm{~h}$ after hCG injection and subjected to parthenogenetic activation by electrical stimulation in combination with 6-DMAP as described above. Activated oocytes were transferred to fertilization medium and cultured for $22-24 \mathrm{~h}$. They were then transferred to mR1ECM drops and culture was continued. Five replicate experiments were performed.

Experiment 3 was designed to test the effect of culture duration on parthenogenetic activation of rat oocytes. Before activation, oocytes were cultured in fertilization medium for $0,2,4$ or $6 \mathrm{~h}$ after collection. Then they were activated by electrical stimulation in combination with 6-DMAP as described above. After activation, the oocytes were transferred to fertilization medium and cultured for 22-24 h. They were then transferred to mR1ECM medium and culture was continued. Three replicate experiments were performed.

Experiment 4 was designed to test the development of oocytes activated by electrical stimulation or ionomycin in combination with 6DMAP. Based on the results of experiments 2 and 3 , oocytes were collected $18 \mathrm{~h}$ after hCG injection and activated immediately by electrical stimulation or incubation in fertilization medium containing 5 $\mu \mathrm{M}$ ionomycin (Sigma) for $5 \mathrm{~min}$, both followed by treatment in fertilization medium containing $2 \mathrm{mM}$ 6-DMAP for $4 \mathrm{~h}$. Then they were cultured in fertilization medium for 22-24 h and in mR1ECM for a further 4 days. Three replicate experiments were performed.

Experiment 5 was designed to test the effect of the osmolarity of the activation medium on the activation and development of oocytes activated by ionomycin in combination with 6-DMAP. Rat oocytes were activated as follows: 1$)$ ionomycin (5 $\mu \mathrm{M}, 5 \mathrm{~min})$ and 6-DMAP ( $2 \mathrm{mM}, 4 \mathrm{~h})$, both in mR1ECM medium ( $\mathrm{mR} 1 \mathrm{ECM}+110 \mathrm{mM}$ $\mathrm{NaCl}+\mathrm{PVA}, 310 \mathrm{mOsM}$ ) and culture in fertilization medium (mR1ECM+110 mM NaCl+BSA, 310 mOsM); 2) ionomycin and 6-DMAP, both in mR1ECM medium (mR1ECM+110 mM $\mathrm{NaCl}+\mathrm{PVA}, 310 \mathrm{mOsM}$ ) and culture in mR1ECM medium (mR1ECM+76.7 mM NaCl, $246 \mathrm{mOsM}$ ) after activation; 3 ) ionomycin in $\mathrm{mR} 1 \mathrm{ECM}$ medium (310 mOsM) and 6-DMAP in mR1ECM medium (246 $\mathrm{mOsM}$ ) and culture in mR1ECM medium (246 $\mathrm{mOsM}$ ) after activation; 4) ionomycin in mR1ECM medium (246 mOsM) and 6-DMAP in mR1ECM medium (310 $\mathrm{mOsM})$ and culture in $\mathrm{mR} 1 \mathrm{ECM}$ medium (246 mOsM) after activation; and 5) ionomycin and 6-DMAP, both in mR1ECM medium (246 mOsM) and culture in $\mathrm{mR} 1 \mathrm{ECM}$ medium (246 mOsM) after activation. Three replicate experiments were performed.

\section{Statistical analysis}

All data are presented as the mean \pm SEM. Data in Tables 1 and 4 were analyzed by student's $t$-test and others by ANOVA followed by Fisher's Least Significant Difference test as a multiple-comparison test after arcsine transformation. Differences with $\mathrm{P}<0.05$ were considered statistically significant.

\section{Results}

Experiment 1. Effect of rat strain on parthenogenetic activation of rat oocytes in vitro

Although there was no significant difference between Wistar-Imamichi and SD strains in the percentages of oocytes activated by electrical stimulation in combination with 6-DMAP treatment and developed to pronuclei $(\mathrm{PN})$, the two-cell, four-cell, and morula stages, the percentage of activated oocytes that developed to the blastocyst was significantly higher in the Wistar-Imamichi rats than in the SD strain $(\mathrm{P}<0.05$, Table 1). 
Table 1. Effects of rat strain on parthenogenetic activation in rat oocytes

\begin{tabular}{lcccccc}
\hline \multirow{2}{*}{$\begin{array}{l}\text { Rat } \\
\text { strains }\end{array}$} & \multicolumn{5}{c}{ No. of oocytes treated and developing to various stages } \\
\cline { 2 - 7 } & Total & PN $(\%)^{*}$ & 2-cells $(\%)^{*}$ & 4-cells $(\%)^{*}$ & Morula(\%) & Bl $(\%)^{*}$ \\
\hline SD & 63 & $60(95.2 \pm 0.2)$ & $42(66.7 \pm 31.7)$ & $16(25.4 \pm 10.4)$ & $8(12.7 \pm 14.6)$ & $2(3.2 \pm 5.9)^{\mathrm{a}}$ \\
WI & 55 & $54(98.2 \pm 3.8)$ & $51(92.7 \pm 4.5)$ & $24(43.6 \pm 11.4)$ & $20(36.4 \pm 8.6)$ & $20(36.4 \pm 8.6)^{\mathrm{b}}$ \\
\hline
\end{tabular}

PN: Pronuclear formation.

* Percentage of the total number of oocytes treated.

Bl: Blastocysts.

SD: Sprague Dawley; WI: Wistar-Imamichi.

a-b Values with different superscripts within the same column are significantly different $(\mathrm{P}<0.05)$.

Table 2. Effects of post-ovulation duration on parthenogenetic activation in rat

\begin{tabular}{lccccc}
\hline \multirow{2}{*}{$\begin{array}{l}\text { Hours post-hCG } \\
\text { injection }\end{array}$} & \multicolumn{4}{c}{ No. of oocytes treated and developing to various stages } \\
\cline { 2 - 6 } & \multicolumn{1}{c}{ Total } & \multicolumn{1}{c}{$\mathrm{PN}(\%)^{*}$} & 2-cells $(\%)^{*}$ & \multicolumn{1}{c}{$\mathrm{Bl}(\%)^{*}$} \\
\hline 16 & 68 & $52(77 \pm 7.0)^{\mathrm{a}}$ & $40(59 \pm 11.2)^{\mathrm{a}}$ & 6 & $(9 \pm 6.0)^{\mathrm{a}}$ \\
18 & 60 & $60(100 \pm 0)^{\mathrm{b}}$ & $54(90 \pm 12.7)^{\mathrm{b}}$ & $25(42 \pm 8.0)^{\mathrm{b}}$ \\
20 & 129 & $128(99 \pm 1.9)^{\mathrm{b}}$ & $120(93 \pm 9.0)^{\mathrm{b}}$ & $59(46 \pm 26.0)^{\mathrm{b}}$ \\
22 & 130 & $128(98 \pm 2.0)^{\mathrm{b}}$ & $111(85 \pm 11.0)^{\mathrm{ab}}$ & $28(22 \pm 14.0)^{\mathrm{c}}$ \\
24 & 85 & $83(98 \pm 6.0)^{\mathrm{b}}$ & $62(73 \pm 17.0)^{\mathrm{ab}}$ & $27(32 \pm 13.0)^{\mathrm{bc}}$ \\
26 & 41 & $38(93 \pm 8.0)^{\mathrm{b}}$ & $27(66 \pm 34.0)^{\mathrm{ab}}$ & $8(20 \pm 1.8)^{\mathrm{c}}$ \\
\hline
\end{tabular}

PN: Pronuclear formation.

* Percentage of the total number of oocytes treated.

Bl: Blastocysts.

a-c Values with different superscripts within the same column are significantly different $(\mathrm{P}<0.05)$.

Experiment 2. Effect of post-ovulation duration on parthenogenetic activation of rat oocytes in vitro

When oocytes were collected $18,20,22,24$ or $26 \mathrm{~h}$ after hCG injection, the percentages of activated oocytes with PN were significantly higher than when they were collected $16 \mathrm{~h}$ post-hCG. The percentages of activated oocytes that developed to the two-cell and blastocyst stages were higher when oocytes were collected at 18 and $20 \mathrm{~h}$ than at other time points after hCG injection $(\mathrm{P}<0.05$, Table 2).

\section{Experiment 3. Effect of culture duration on parthenogenetic activation of rat oocytes}

As shown in Table 3, culturing the oocytes in fertilization medium for $0-6 \mathrm{~h}$ before activation treatment did not affect the activated oocytes that developed to pronuclei and the two-cell stage. However, the percentages of activated oocytes that developed to the four-cell, morula and blastocyst stages were significantly higher when oocytes were subjected to activation treatment immediately $(0 \mathrm{~h})$.
Experiment 4. The development of oocytes activated by electrical stimulation or ionomycin treatment in combination with 6-DMAP

There were no significant differences in the percentages of activated oocytes with $\mathrm{PN}$ and those developed to the two-cell, four-cell, morula and blastocyst stages between two treatments (ionomycin with 6-DMAP vs electrical stimulation with 6-DMAP) (Table 4).

Experiment 5. Effects of osmolarity of activation medium on parthenogenetic activation and subsequent development of rat oocytes

Table 5 shows that culturing activated oocytes in fertilization medium for 22-24 h (310 mOsM) after activation did not improve subsequent development (Groups A and B). Therefore, oocytes were cultured directly in mR1ECM culture medium (246 mOsM) for development after activation of the C-E treatment groups. After treatment with ionomycin, culturing oocytes in 6-DMAP solution with low osmolarity (246 mOsM) significantly increased the percentages of activated oocytes that 
Table 3. Effects of culture duration on parthenogenetic activation in rat oocytes

\begin{tabular}{|c|c|c|c|c|c|c|}
\hline \multirow{2}{*}{$\begin{array}{l}\text { Culture } \\
\text { duration } \\
\text { (h) }\end{array}$} & \multirow[b]{2}{*}{ Total } & \multicolumn{5}{|c|}{ No. of oocytes treated and developing to various stages } \\
\hline & & $\mathrm{PN}(\%)^{*}$ & 2 -cells $(\%)^{*}$ & 4 -cells $(\%)^{*}$ & Morula $(\%)^{*}$ & $\mathrm{Bl}(\%)^{*}$ \\
\hline 0 & 38 & $37(97.4 \pm 5.7)$ & $36(94.7 \pm 3.8)$ & $31(81.6 \pm 9.3)^{\mathrm{a}}$ & $30 \quad(78.9 \pm 12.2)^{\mathrm{a}}$ & $23(60.5 \pm 12.5)^{\mathrm{a}}$ \\
\hline 2 & 47 & $47 \quad(100 \pm 0)$ & $45(95.7 \pm 4.3)$ & $31(66.0 \pm 9.0)^{\mathrm{ab}}$ & $25(53.2 \pm 9.4)^{\mathrm{b}}$ & $17(36.2 \pm 13.8)^{\mathrm{ab}}$ \\
\hline 4 & 45 & $45 \quad(100 \pm 0)$ & $41(91.1 \pm 4.4)$ & $22(48.9 \pm 11.1)^{\mathrm{bc}}$ & $18(40.0 \pm 13.3)^{b c}$ & $7(15.6 \pm 8.9)^{\mathrm{bc}}$ \\
\hline 6 & 46 & $44(95.4 \pm 7.9)$ & $40(86.9 \pm 6.9)$ & $19(41.3 \pm 8.0)^{\mathrm{c}}$ & $12(26.1 \pm 6.1)^{c}$ & $4 \quad(8.7 \pm 10.0)^{c}$ \\
\hline
\end{tabular}

PN: Pronuclear formation.

*Percentage of the total number of oocytes treated.

Bl: Blastocysts.

${ }^{a-c}$ Values with different superscripts within the same column are significantly different $(\mathrm{P}<0.05)$.

Table 4. Development in vitro of rat oocytes activated by electrical stimulation (ES) or ionomycin (Ionomycin) in combination with 6-DMAP

\begin{tabular}{|c|c|c|c|c|c|c|}
\hline \multirow{2}{*}{$\begin{array}{l}\text { Activation } \\
\text { method }\end{array}$} & \multirow[b]{2}{*}{ Total } & \multicolumn{5}{|c|}{ No. of oocytes treated and developing to various stages } \\
\hline & & $\mathrm{PN}(\%)^{*}$ & 2 -cells $(\%)^{*}$ & 4 -cells $(\%)^{*}$ & Morula $(\%)^{*}$ & $\mathrm{Bl}(\%)^{*}$ \\
\hline $\mathrm{ES}+\mathrm{D}$ & 38 & $(96.7$ & $23(66.3$ & $18(51.7=$ & $18(51.7$ & $12(42.3$ \\
\hline Ionomycin+DMAP & 32 & $31(96.7 \pm 3.3)$ & $24(77.3 \pm 17.9)$ & $17(55.7 \pm 19.5)$ & $14(45.7 \pm 15.5)$ & $13(42.7 \pm 18.3)$ \\
\hline
\end{tabular}

PN: Pronuclear formation.

* Percentage of the total number of oocytes treated.

Bl: Blastocysts.

Table 5. Effect of osmolarity of activation media on parthenogenetic activation of rat oocytes treated by ionomycin and 6-DMAP

\begin{tabular}{|c|c|c|c|c|c|c|c|c|}
\hline \multirow[b]{2}{*}{ Group } & \multicolumn{3}{|c|}{ Activation media (mOsM) } & \multicolumn{5}{|c|}{ No. of oocytes treated and developing to various stages } \\
\hline & $\mathrm{I}$ & $\mathrm{D}$ & FM & Total & 2 -cells $(\%)^{*}$ & 4 -cells $(\%)^{*}$ & Morula $(\%)^{*}$ & $\mathrm{Bl}(\%)^{*}$ \\
\hline A & 310 & 310 & + & 52 & $43(83.0 \pm 5.7)^{\mathrm{a}}$ & $26(49.7 \pm 5.8)^{\mathrm{a}}$ & $25(48.0 \pm 5.3)^{\mathrm{a}}$ & $13(25.3 \pm 3.2)^{\mathrm{a}}$ \\
\hline B & 310 & 310 & - & 48 & $32(66.7 \pm 8.3)^{\mathrm{a}}$ & $26(54.3 \pm 5.6)^{\mathrm{a}}$ & $24(50.3 \pm 7.2)^{\mathrm{a}}$ & $11(23.0 \pm 5.3)^{\mathrm{a}}$ \\
\hline $\mathrm{C}$ & 310 & 246 & - & 31 & $28(90.7 \pm 5.2)^{b}$ & $26(84.0 \pm 3.1)^{\mathrm{bc}}$ & $17(55.3 \pm 7.4)^{\mathrm{a}}$ & $11(35.3 \pm 2.9)^{\mathrm{a}}$ \\
\hline $\mathrm{D}$ & 246 & 310 & - & 48 & $34(71.0 \pm 11.2)^{\mathrm{a}}$ & $32(66.0 \pm 9.0)^{\mathrm{ac}}$ & $25(52.3 \pm 5.6)^{\mathrm{a}}$ & $11(23.3 \pm 7.5)^{\mathrm{a}}$ \\
\hline E & 246 & 246 & - & 51 & $50(98.0 \pm 2.0)^{\mathrm{b}}$ & $49(96.3 \pm 1.9)^{\mathrm{d}}$ & $48(94.0 \pm 0.6)^{\mathrm{b}}$ & $36(68.7 \pm 10.7)^{b}$ \\
\hline
\end{tabular}

I: mR1ECM supplemented with $5 \mu \mathrm{M}$ ionomycin.

D: mR1ECM supplemented with 2 mM 6-DMAP.

FM: Fertilization medium supplemented with BSA (310 mOsM).

* Percentage of the total number of oocytes treated.

Bl: Blastocysts.

a-d Values with different superscripts within the same column are significantly different $(\mathrm{P}<0.05)$.

developed to the two-cell and four-cell stages, but not the percentages that developed to morula and blastocysts (Group C). Activation of oocytes in ionomycin solution with low osmolarity (246 mOsM) did not affect the cleavage rates (Group D). However, treatment of oocytes with ionomycin and 6-DMAP, both in low osmolarity solutions (246 $\mathrm{mOsM})$, markedly increased the percentages of activated oocytes that developed not only to the two- to four-cell stages, but also to blastocysts (Group $\mathrm{E}, \mathrm{P}<0.05)$. The treatments of this group also improved the quality of blastocysts, resulting in good morphology and large cell numbers (42.8 \pm 2.4, Fig. 1).

\section{Discussion}

The results of the present study show that oocytes from WI and SD rat strains respond differently to activation by electrical stimulation in combination with 6-DMAP treatment. In addition, 

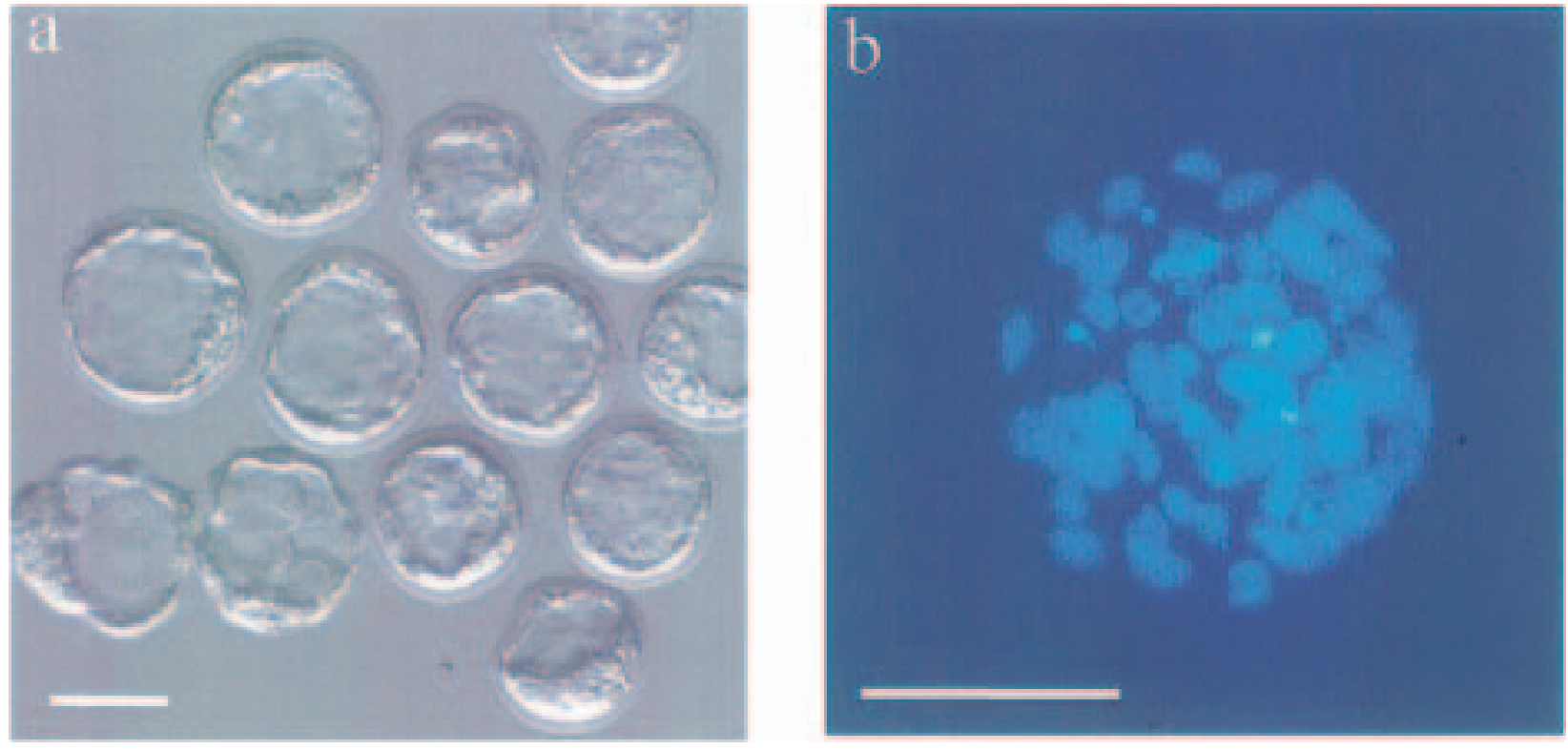

Fig. 1. Morphology (a) and cell number (b) of blastocysts developed from rat oocytes activated by ionomycin and 6-DMAP. Bars $=100 \mu \mathrm{m}$.

the optimal conditions determined for parthenogenetic activation and subsequent development in WI rats are as follows: oocyte collection $18 \mathrm{~h}$ post-hCG injection, and immediate subjection to activation treatment by ionomycin ( 5 $\mu \mathrm{M}, 5 \mathrm{~min}$ ) in combination with 6-DMAP ( $2 \mathrm{mM}, 4$ $\mathrm{h})$, both in low-osmolarity mR1ECM, followed by and direct culture in culture medium R1ECM for subsequent development. These conditions made the activation procedure simple and efficient, as indicated by the high rate of development of blastocysts with good morphology and large cell numbers.

Genetic background affects parthenogenetic activation in mice $[14,15]$. A study using several rat strains showed that the developmental potential in culture (mR1ECM) of fertilized rat eggs is straindependent. A larger proportion of fertilized 1-cell embryos $(76 \%)$ from outbred SD rats developed to the blastocyst stage than those $(0-12 \%)$ from inbred strains, including WF, LEW, F544, PVG [3]. These findings suggest that genetic background in rats also affects the oocyte response in vitro. In fact, the finding of Kato et al. (2001) showed that oocytes from Wistar and SD rats had different responses to parthenogenetic activation induced by strontium treatment. When oocytes from SD or Wistar rats were incubated with $1.25 \mathrm{mM}$ strontium for $6 \mathrm{~h}$, the oocytes from the SD strain were less sensitive to the strontium treatment than the oocytes from the Wistar strain (survival rate 99 vs $87 \%$ ). The overall activation efficiency for SD oocytes was higher than that for Wistar oocytes (74 vs $45 \%$ ) [6]. However, the results of the present study indicate that although there was no difference in the percentages of activated oocytes with pronuclei between SD and WI rats (95 vs $98 \%$ ), blastocyst formation rates were markedly higher in the WI than in the SD strain when oocytes were activated by electrical stimulation in combination with 6-DMAP treatment. These findings suggest that parthenogenetic activation and subsequent development of rat oocytes are dependent not only on genetic background, but also on the treatment procedures applied.

Parthenogenetic activation of oocytes depends on age and species. It has been shown that aged oocytes are more sensitive to activation treatment in vitro in mice [16], cows [17, 18] and rabbits [19]. Although aged and young porcine oocytes after activation had the same developmental potential in vitro [20], the developmental ability of activated bovine oocytes decreased with the age of the oocytes [17]. Information on the effect of oocyte age on the response to activation treatment in the rat is very limited. A study in Wistar rats [8] showed that the use of ethanol for parthenogenetic activation was highly effective only for aged 
oocytes (22-24 h after hCG). However, the efficiencies of activation and cleavage rate were equal for all ages of oocytes when they were treated with strontium [8]. The results of the present study show that when WI rat oocytes were activated by electrical stimulation followed by 6-DMAP, higher percentages of treated oocytes developed to the two-cell and blastocyst stages when oocytes were collected at 18 or $20 \mathrm{~h}$ than at other time points after hCG injection $(\mathrm{P}<0.05)$.

Ovulated rat oocytes have been reported to become activated spontaneously during in vitro culture $[6,21]$. This specific characteristic may affect the results of the activation treatment in vitro. A recent study indicated that SD rat oocytes activated $10 \mathrm{~min}$ or $2 \mathrm{~h}$ following cervical dislocation showed higher blastocyst development than those of the 4 and 6 h groups [22]. Our results using WI rat oocytes in the present study show that although there was no difference in the percentages of activated oocytes with pronuclei, the ability to develop to the blastocyst stage declined significantly as the culture duration before activation was increased. A significantly higher percentage of blastocysts $(60.5 \%)$ was obtained when oocytes were immediately subjected to activation treatment $(0 \mathrm{~h})$ than when they were cultured for $2-6 \mathrm{~h}$ after collection $(8.7-36.2 \%$, $\mathrm{P}<0.05)$. These results suggested that timing of activation might be an important factor in rat clone production.

In a previous study, we reported that the combination of electrical stimulation with 6-DMAP markedly improved parthenogenetic activation and subsequent development of rat oocytes to the blastocyst stage in vitro [10]. However, the percentage of blastocysts developed from activated rat oocytes was still low and the quality of blastocysts obtained was poor. In an attempt to simplify the activation procedure and improve the activation efficiency and quality of blastocysts in the present study, we compared two protocols for inducing oocyte activation. Our present results show that the cleavage and blastocyst formation rates tended to be higher when oocytes were activated by ionomycin combined with 6-DMAP treatment than by electrical stimulation combined with 6-DMAP treatment although there were no significant differences between the two treatments. Based on these results and the fact that it is much simpler to treat oocytes with ionomycin than electrical stimulation, ionomycin combined with 6DMAP treatment was used to determine the effect of the osmolarity of the activation medium on parthenogenetic activation and subsequent development of WI rat oocytes. It has been reported that the osmolarity of the medium influenced in vitro fertilization and subsequent development of rat oocytes. High osmolarity (310 $\mathrm{mOsM}$ ) in the medium resulting from an elevated sodium chloride concentration $(110 \mathrm{mM})$ improved pronuclear formation and subsequent development when rat oocytes were fertilized in vitro [11]. In contrast, the results of the present study demonstrate that a markedly higher percentage and quality of blastocysts were obtained when rat oocytes were activated by ionomycin in combination with DMAP, both in low-osmolarity (246 mOsM) medium (69\%), than in any other group $(23-35 \%, \mathrm{P}<0.05)$. It has been reported that rat parthenogenetic blastocysts had mean cell numbers as low as 24.9 [8] to 26.9 [10]. The mean cell number of blastocysts (42.8) obtained in the present study was much higher than those of previous reports, and is comparable with those of blastocysts developed from fertilized oocytes cultured in vitro $(45.8 \pm 3)$. In addition, similar results with high percentages of oocytes developed to the blastocyst stage were obtained when activation was induced by ionomycin, DMAP and cytochalasin B (for diploidization) (data not shown).

In conclusion, we have developed a simple and effective method for inducing parthenogenetic activation of WI rat oocytes, which resulted in a much higher percentage and quality of blastocysts obtained than has been previously reported. These findings may be helpful for nuclear transfer in rats.

\section{Acknowledgments}

The authors thank Dr. Teruhiko Wakayama (Laboratory of Genomic Reprogramming, Center for Developmental Biology, RIKEN Kobe Institute, Kobe, Hyogo, Japan) for critical reading of the manuscript.

This work was supported by grants from the Program for Promotion of Basic Research Activities for Innovative Biosciences and "Research for the Future" Program, the Japan Society for the Promotion of Science (JSPS-RFTF97L00904). 


\section{References}

1. Galli C, Lagutina I, Crotti G, Colleoni S, Turini P, Ponderato N, Duchi R, Lazzari G. Pregnancy: A cloned horse born to its dam twin. Nature 2003; 424: 635.

2. Jiang JY, Mizuno S, Mizutani E, Miyoshi K, Kimura N, Sasada H, Sato E. Nuclear transfer in rats using an established embryonic cell line and cumulus cells. J Reprod Dev 2002; 48: 505-511.

3. Iannaccone $\mathbf{P}$, Taborn G, Garton $\mathbf{R}$. Preimplantation and postimplantation development of rat embryos cloned with cumulus cells and fibroblasts. Zygote 2001; 9: 135-143.

4. Fitchev P, Taborn G, Garton $\mathbf{R}$, Iannaccone $\mathbf{P}$. Nuclear transfer in the rat: potential access to the germline. Transplant Proc 1999; 31: 1525-1530.

5. Hayes E, Galea S, Verkuylen A, Pera M, Morrison J, Lacham-Kaplan O, Trounson A. Nuclear transfer of adult and genetically modified fetal cells of the rat. Physiol Genomics 2001; 5: 193-204.

6. Kato M, Hirabayashi M, Aoto T, Kazumi I, Ueda M, Hochi S. Strontium-induced activation regimen for rat oocytes in somatic cell nuclear transplantation. J Reprod Dev 2001; 47: 407-413.

7. Zhou Q, Renard JP, Le Friec G, Brochard V, Beaujean N, Cherifi Y, Fraichard A, Cozzi J. Generation of fertile cloned rats by regulating oocyte activation. Science 2003; 302: 1179.

8. Krivokharchenko A, Popova E, Zaitseva I, Vil'ianovich L, Ganten D, Bader M. Development of parthenogenetic rat embryos. Biol Reprod 2003; 68: 829-836.

9. Zernicka-Goetz M. Spontaneous and induced activation of rat oocytes. Mol Reprod Dev 1991; 28: 169-176.

10. Jiang JY, Mizuno S, Mizutani E, Sasada H, Sato E. Parthenogenetic activation and subsequent development of rat oocytes in vitro. Mol Reprod Dev 2002; 61: 120-125.

11. Oh SH, Miyoshi K, Funahashi H. Rat oocytes fertilized in modified rat 1-cell embryo culture medium containing a high sodium chloride concentration and bovine serum albumin maintain developmental ability to the blastocyst stage. Biol Reprod 1998; 59: 884-889.
12. Miyoshi K, Kono T, Niwa K. Stage-dependent development of rat 1-cell embryos in a chemically defined medium after fertilization in vivo and in vitro. Biol Reprod 1997; 56: 180-185.

13. Miyoshi K, Abeydeera LR, Okuda K, Niwa K. Effects of osmolarity and amino acids in a chemically defined medium on development of rat one-cell embryos. J Reprod Fertil 1995; 103: 27-32.

14. Marcus GJ. Activation of cumulus-free mouse oocytes. Mol Reprod Dev 1990; 26: 159-162.

15. Rybouchkin A, Dozortsev D, de Sutter PD, Dhont M. Factors affecting the role of the spindle during early response of mouse oocytes to ethanol stimulation. J Exp Zool 1996; 275: 469-475.

16. Otaegui PJ, O'Neill GT, Wilmut I. Parthenogenetic activation of mouse oocytes by exposure to strontium as a source of cytoplasts for nuclear transfer. Cloning 1999; 1: 111-117.

17. Susko-Parrish JL, Leibfried-Rutledge ML, Northey DL, Schutzkus V, First NL. Inhibition of protein kinases after an induced calcium transient causes transition of bovine oocytes to embryonic cycles without meiotic completion. Dev Biol 1994; 166: 729_ 739.

18. Presicce GA, Yang $\mathbf{X}$. Nuclear dynamics of parthenogenesis of bovine oocytes matured in vitro for 20 and 40 hours and activated with combined ethanol and cycloheximide treatment. Mol Reprod Dev 1994; 37: 61-68.

19. Collas P, Robl JM. Factors affecting the efficiency of nuclear transplantation in the rabbit embryo. Biol Reprod 1990; 43: 877-884.

20. Jolliff WJ, Prather RS. Parthenogenetic development of in vitro-matured, in vitro-cultured porcine oocytes beyond blastocysts. Biol Reprod 1997; 56: 544-548.

21. Keefer CL, Schuetz AW. Spontaneous activation of ovulated rat oocytes during in vitro culture. J Exp Zool 1982; 224: 371-377.

22. Roh S, Malakooti N, Morrison JR, Trounson AO, Du ZT. Parthenogenetic activation of rat oocytes and their development (in vitro). Reprod Fertil Dev 2003; 15: 135-140. 